\title{
THE DAMPING OF WAVES AND OTHER DISTURB- ANCES IN MERCURY
}

\author{
By M. H. Stillman, Assistant Physicist
}

In connection with some work on the adjustment of mercury surfaces, done by the writer some time ago, ${ }^{1}$ considerable difficulty was experienced by reason of the small waves that formed on the surface of the mercury as a.result of the trembling of the support of the vessel containing the mercury. The present paper describes a method by which such disturbances of a mercury surface, as well as larger disturbances of the mercury as a whole, can be partially or wholly eliminated.

This method consists in subjecting the mercury to a strong magnetic field, the direction of the field being approximately at right angles to the direction of motion or of impending motion of some portion of the mercury. The motion of the mercury across the magnetic lines of force tends to produce an electric current, the reaction of which with the lines of force tends to stop the motion of the mercury.

Let $\quad l=$ the effective length of an element of mercury which is cutting the magnetic lines of force at right angles and the axis of which is at right angles to the lines of force.

$v=$ the velocity of the element of mercury in a direction at right angles to the lines of force and to the element. 
$E=$ the electromotive force produced in the element by the motion of the mercury relative to the lines of force.

$I=$ the electric current produced in the element by this electromotive force.

$R=$ the electrical resistance of the elementary circuit.

$\phi=$ the number of lines of force linked with the elementary circuit.

$B=$ the flux density; that is, the number of lines of force per unit area taken perpendicular to the direction of the lines of force.

$f=$ the electromagnetic damping force on the element of mercury per unit length.

Now $f=I B$

$$
I=\frac{E}{R} \text { and } E=\frac{d \phi}{d t}
$$

But $\frac{d \phi}{d t}=l v B$

So that $I=\frac{l v B}{R}$

$$
\therefore f=\frac{l v B^{2}}{R}
$$

A constant $K$ is placed before the second member of this equation to indicate that the numerical value is dependent upon the units used. The equation is then written

$$
f=K \frac{l \approx B^{2}}{R}
$$

If $j$ is expressed in dynes, $l$ in centimeters, $i$ in centimeters per second, $B$ in gausses, and $R$ in ohms, $K$ will be $10^{-9}$, so that

$$
f=10^{-9} \frac{l \omega B^{2}}{R}
$$

The total damping force, $F$, is then the sum of the damping forces on all of the elements; that is,

$$
F=10^{-9} \sum \frac{l i B^{2}}{R}
$$

This clearly indicates the methods by which the damping can be varied. 
In order to determine the effectiveness of the method, the experiments described below were performed. Mercury to a depth of about $\mathrm{I} \mathrm{cm}$ was placed in a glass dish which had a diameter of $43 \mathrm{~mm}$ and this was then placed between the poles of an electromagnet, Fig. r. The field of this magnet was horizontal and its intensity was varied between 2500 and ro 000 gausses during the experiments. It was found that if the mercury were violently agitated as a whole while outside of the magnetic field and then brought into the field, the motion was strongly damped. The magnitude of the damping in a field of given strength was, as expected, dependent upon the direction of motion of the mercury relative to the direction of the field. Thus, while violent oscilla-
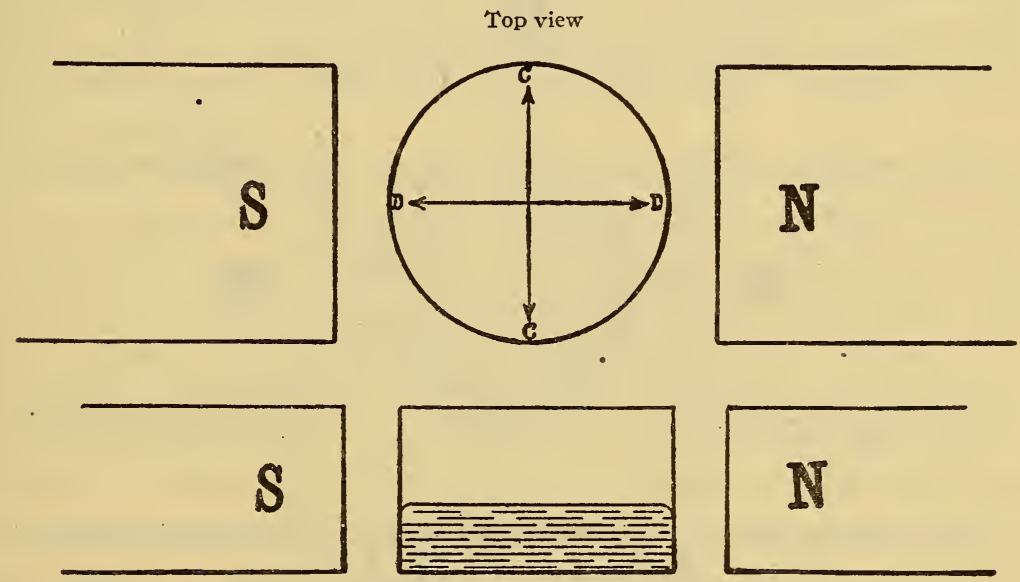

Side view

FIG. I.-Diagram of arrangement of apparatus

tions of the mercury in the direction $C C$ at right angles to the direction of the field persisted for a short time-although brought to rest much sooner than they were outside of the magnetic fieldoscillations in the direction $D D$, parallel to the field, were damped out almost instantly. Keeping the mercury in the field, it was found to be very difficult to produce large oscillations in the direction parallel to $D D$ when the field strength was no greater than approximately 7000 gausses.

It is of some interest and importance to consider the conditions that are responsible for the differences in damping of the oscillations of the mercury in different directions. Fig. 2 represents the conditions existing when the horizontal oscillations of the mercury are at right angles to the lines of force of the field. Fig. 3 repre- 
sents the corresponding conditions when the direction of the horizontal motion is parallel to the direction of the field.

Considering Fig. 2, when the horizontal motion of the mercury as a whole is in the direction shown by the double arrows, an
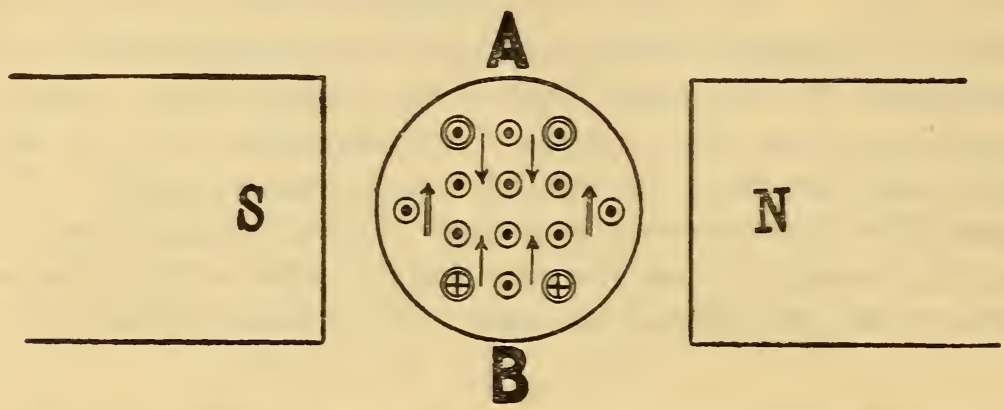

FIG. 2.-Horizontal motion of mercury at right angles to lines of magnetic force Top riew
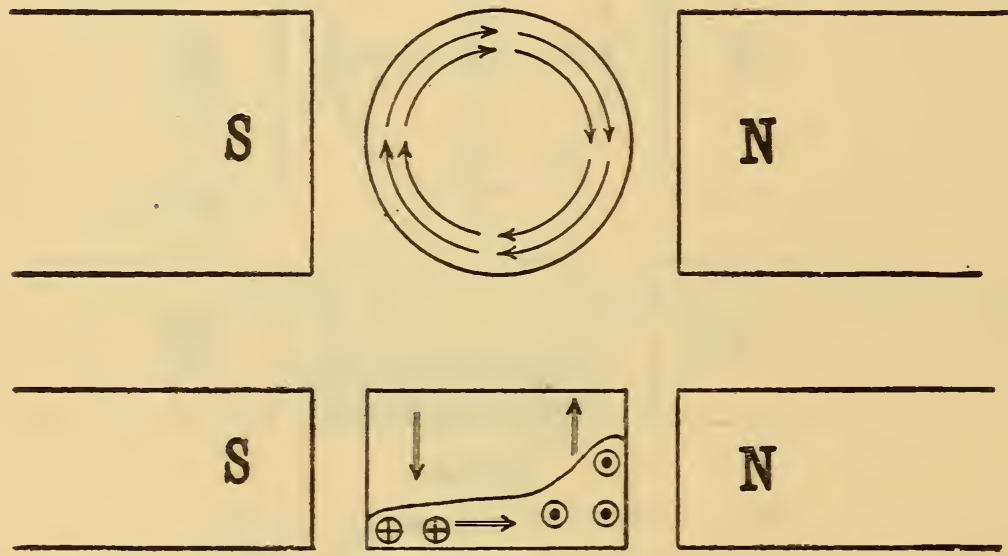

Side view

FIG. 3.-Horizontal motion of mercury in direction of lines of magnetic force

\section{Legend for n̂gures 2 and 3}

$\Longrightarrow$ Direction of motion of the mercury
$\longrightarrow$ Direction of induced emf or current
(9) Mercury rising
Mercury falling
Induced emf or current directed upward or toward observer

uprrard-directed emf is developed in accordance with the laws of electromagnetic induction in all parts of the mercury so moving; but inasmuch as there is no adequate return circuit available, very little current flows and therefore, in accordance with the formula, there is very little damping. In addition to this hori- 
zontal motion of the mercury, there is a vertical upward motion at the far side $(A)$ of the vessel and a corresponding downward motion at the near side $(B)$. This produces two opposing emf's, as illustrated in Fig. 2, so that also by this last-mentioned type of motion little current is produced and consequently the damping is small.

In Fig. 3, which illustrates the conditions existing when the horizontal motion of the mercury is parallel to the lines of force of the field, it is evident that no emf and therefore no current is produced by this horizontal motion. But, as in the type of motion first considered, this horizontal motion is accompanied by a rising of the mercury on one side of the vessel and a corresponding falling on the other side. These movements in opposite directions produce emf's in opposite directions, but instead of opposing, these reinforce each other and hence produce a current as illustrated in the figure.

The fact brought out in the above discussion, namely, that an electric current of an effective magnitude in the mercury is often prevented by opposing electromotive forces or lack of a return circuit of low resistance, suggested that if the containing vessel were of some nonmagnetic metal of low resistance instead of the electrically nonconducting glass, and if the mercury were in good electrical contact with the metal, much greater damping might be expected to occur, especiaily of those oscillations which involve a horizontal movement of the mercury across the lines of force. Accordingly, a brass vessel was constructed, its interior having the same dimensions as that of the glass vessel and having walls about $5 \mathrm{~mm}$ thick. This was supplied with the same quantity of mercury as that contained by the glass vessel and then the oscillations of the mercury in the two vessels were compared, both inside and outside of the magnetic field. As was to be expected, the motion of the mercury in the two vessels when the magnetic field was not present was practically the same. When, however, the magnetic field was present the effectiveness of the metallic container in comparison with the glass container in aiding the magnetic damping of the mercury was very marked. This advantage was especially evident when the horizontal motion of the mercury was perpendicular to the direction of the magnetic field, the time taken to completely damp the oscillations being only a small fraction of that required when the glass vessel was employed.

For continued use a plain brass vessel would of course not be satisfactory because of the tendency of brass and mercury to 
amalgamate. A nickel-plated copper or brass vessel would probably be very successful, combining resistance to amalgamation with low electrical resistance. When a glass containing vessel must be used, a nonmagnetic wire netting of low electrical resistance and not easily amalgamated might be submerged just below the surface of the mercury. This would aid the magnetic field in damping the oscillations by providing the induced electric currents with a return path of high conductivity, and it would also tend to damp these oscillations mechanically.

These above-described experiments refer to the damping out of the relatively large oscillations or other large disturbances in a mass of mercury, and it is this type of disturbance that the method is particularly effective in reducing or obviating, since the damping is directly proportional to the velocity of the mercury. However, the writer has also found that the smaller ripples on the mercury surface may also be strongly damped by the use of the magnetic field. In these experiments it was found that even by the use of the parallel transverse magnetic field used in the previously described experiments-a type of field not especially well adapted to this purpose-the ripples were damped out in less than one-half of the time required for them to subside unaided. By carefully designing the mercury container and the magnetic field, making use of the facts expressed in the equation, the damping of these waves could be much increased.

Attention should be called to the fact that it is not always necessary to expose the entire surface of the mercury to the magnetic field in order to secure this damping effect on the ripples; but, since the damping force varies as the square of the field strength, it would in many instances be more economical to make the field strength large and to limit its operation to a comparatively small area at that portion of the system where the mercury motion and the electrical conductivity are most favorable for damping.

While, as it is almost unnecessary to state, some simpler method than above described may generally be used to damp out the motion in a mass of mercury, it is very probable that this method used by itself or in conjunction with some other method will frequently be found to have distinct advantages. It is suggested that it might sometimes be used when accurate adjustments of mercury surfaces at sea are desired and in other places where unsteadiness of the mercury container is unavoidable.

Washington, April i5, igi6. 